\title{
Tailoring the Response of Silicon Photonics Devices
}

\author{
G. T. Reed, F. Y. Gardes, B. D. Timotijevic, W. R. Headley, and G. Z. Mashanovich \\ Advanced Technology Institute, University of Surrey, \\ Guildford, Surrey, GU2 7XH, UK \\ g.reed@surrey.ac.uk
}

\begin{abstract}
Shrinking waveguide dimensions in silicon photonics results in a series of performance enhancements, but at some cost. We analyse the waveguide geometry in optical modulators and filters to address some issues associated with this trend.
\end{abstract}

Silicon photonics has experienced rapid development for the last three years and several significant results have been reported, demonstrating the viability of the technology [e.g., 1, 2, 3, 4]. One of the recent trends in silicon photonics has been the reduction of waveguide dimensions. This reduction facilitates tighter bending radii and therefore a smaller device footprint which in turn, significantly reduces the cost. Furthermore, technical performance of many silicon photonic devices is enhanced. However, there are also some issues with this trend to smaller dimensions, notably increased propagation losses, increased polarisation dependence and difficulty in coupling to/from optical fibres. These issues can be overcome with a careful design of the waveguide and device geometry.

We have previously shown that by reducing waveguide dimensions, multi-GHz bandwidth optical modulators can be achieved by utilising a horizontal pn junction in a waveguide with an overall height of $450 \mathrm{~nm}$ [5]. Although the waveguide structure of these previous devices has been designed to approach birefringence-free propagation, the fact that the pn junction is horizontal introduces a difference between the phase shifts for TE and TM polarisations, and hence the device itself is not polarisation independent.

In order to improve the polarisation performance to approach polarisation independence of the modulator, we propose here a modulator with a pn junction that is a V-shape structure, as shown in Figure 1. The V-shape is based on the natural etch angle of silicon (54.7 degrees). The operation of the modulator is similar to that presented in [5] in that it is based upon a pn junction. If the rib waveguide is polarisation independent, by varying the height of the $\mathrm{V}$-shaped pn junction in the rib, it is now possible to achieve an identical phase shift for TE and TM polarisations.

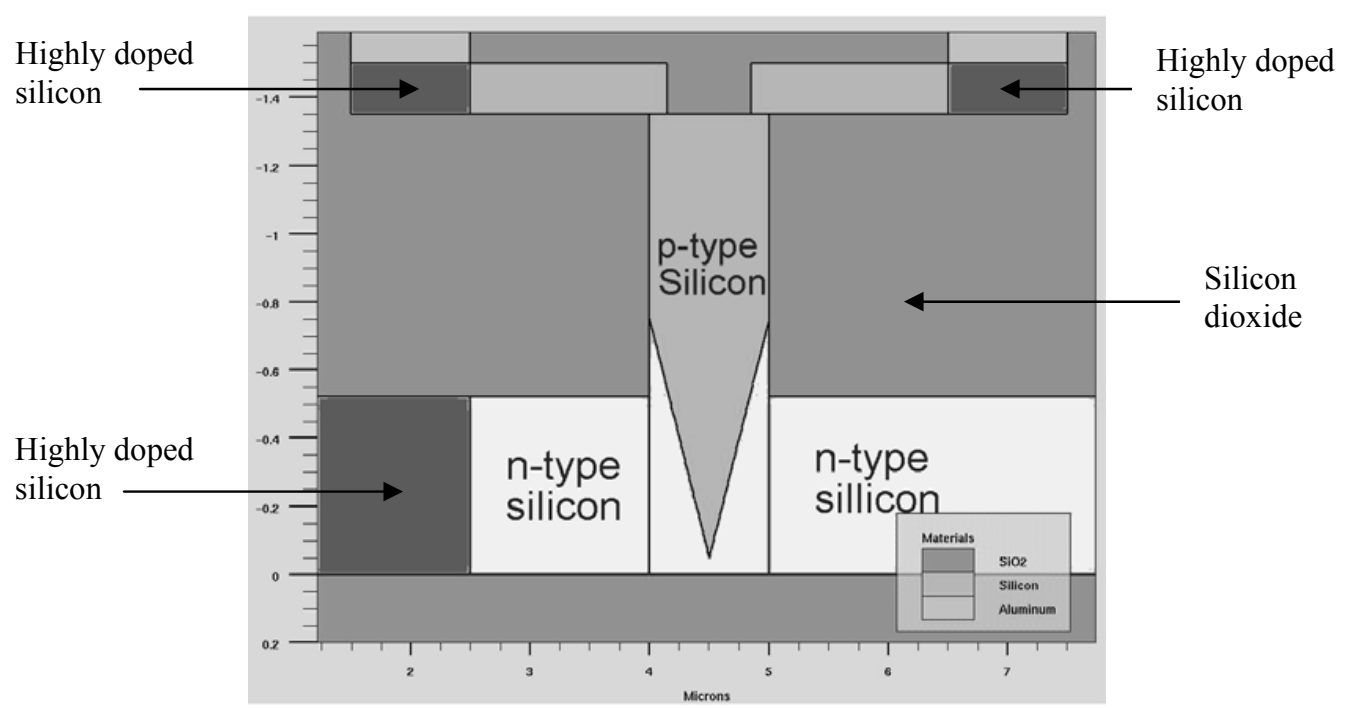

Figure 1. V-shape pn junction optical modulator in silicon technology 
The proposed device is a four terminal diode and is operated by applying a reverse bias. The p-type silicon is situated in the top region of the rib waveguide, and the n-type region is in the slab region. The angled V-shape increases the effective length of the pn junction as well as allowing it to affect TE and TM polarisations in a similar way. Because the natural etch angle of silicon is not $45^{\circ}$, the additional flexibility of vertical adjustment of the V-structure allows sufficient flexibility to equalise the TE and $\mathrm{TM}$ phase responses. The doping profiles of the $\mathrm{p}$ and $\mathrm{n}$ regions facilitate an increased depletion of the p-type region compared to the n-type, in the centre of the optical mode, maximising the change in effective index. Consequently, the concentration of n-type carriers in the waveguide can be reduced, hence decreasing the losses in the waveguide.

Reducing device dimensions can also be beneficial for other devices. An obvious example is the ring resonator based optical filter. It is also clear that rib waveguides offers better control for satisfying both single mode and polarisation independent propagation [6]. We have already shown that polarisation independent ring resonator filters based upon relatively small rib waveguides can be achieved [7]. However, the main limitation of a rib waveguide with cross sectional dimensions of the order of $1 \mu \mathrm{m}$, is loss in resonator bends when the radius of the curvature is of the order of $10 \mu \mathrm{m}$ or less. This is a significant restriction because maximising the Free Spectral Range (FSR), which is of great importance in filters, requires a small device circumference and, therefore, bend radii as small as possible.

Strip waveguides (or silicon wires) allow very small bend radii to be used resulting in a compact footprint, but they suffer from increased loss and significant polarisation issues. Lack of a platform and design which would guarantee both desirable modal and polarisation properties has resulted in research in the area of optical filters evolving in two directions - small strip waveguide based filters and relatively large rib waveguide based filters.

We have previously employed multi-stage ring configurations based upon rib waveguides to increase FSR, FWHM and finesse of the resonator. By using Vernier configurations it is possible to achieve FSR of $\sim 12 \mathrm{~nm}$ [8]. On the other hand, small strip waveguides allow bend radii of 1-3 $\mu \mathrm{m}$, and for a single resonator we have measured an FSR of $47 \mathrm{~nm}$ [9]. A Vernier device based on silicon wires can achieve significantly larger FSR.

\section{References}

[1] L. Liao, D. Samara-Rubio, M. Morse, A. Liu, D. Hodge, D. Rubin, U. D. Keil, and T. Franck, "High speed silicon Mach-Zehnder modulator," Opt. Express, vol. 13, pp. 3129-3135, 2005.

[2] H. Rong, A. Liu, R. Jones, O. Cohen, D. Hak, R. Nicolaescu, A. Fang, and M. Paniccla, "An allsilicon Raman laser," Nature, vol. 433, pp. 292-294, 2005.

[3] Y.-H. Kuo, Y. K. Lee, Y. Ge, S. Ren, J. E. Roth, T. I. Kamins, D. A. B. Miller, and J. S. Harris, "Strong quantum-confined Stark effect in germanium quantum-well structures on silicon," Nature, vol. 437, pp. 1334-1336, 2005.

[4] J. Yongqiang, J. Wei, G. Lanlan, C. Xiaonan, and R. T. Chen, "80-micron interaction length silicon photonic crystal waveguide modulator," Appl. Phys. Lett., vol. 87, pp. 221105-7, 2005.

[5] F. Y. Gardes, G. T. Reed, N. G. Emerson, and C. E. Png, "A sub-micron depletion-type photonic modulator in silicon on insulator," Opt. Express, vol. 13, pp. 8845-8854, 2005.

[6] S. P. Chan, C. E. Png, S. T. Lim, V. M. N. Passaro, and G. T. Reed, "Single mode and polarisation independent SOI waveguides with small cross section," J. Lightwave Technol., vol. 23, 1573-1582, 2005.

[7] W. R. Headley, G. T. Reed, M. Paniccia, A. Liu, O. Cohen, and D. Hak, "Polarization-independent optical racetrack resonators using rib waveguides on silicon-on-insulator," Appl. Phys. Lett., vol. 85, 5523-5525, 2004.

[8] G. T. Reed, G. Z. Mashanovich, W. R. Headley, S. P. Chan, B. D. Timotijevic, and F. Y. Gardes, "Silicon photonics: are small devices always better", Jap. J. Appl. Phys., (in press) 2006.

[9] G. T. Reed, W. R. Headley, F. Y. Gardes, B. D. Timotijevic, S. P. Chan, and G. Z. Mashanovich, "Characteristics of rib waveguide racetrack resonators in SOI," Proc. SPIE, vol. 6183, 2006. 